\title{
Manufatura Aditiva: o papel da soldagem nesta janela de oportunidade
}

\author{
(Additive Manufacturing: the role of welding in this window of opportunity)
}

\author{
Eduardo André Alberti', Leandro João da Silva', Ana Sofia C. M. d'Oliveira ${ }^{2}$ \\ ${ }^{1}$ PGMec, Universidade Federal do Paraná, Curitiba, Paraná, Brasil, edualberti@outlook.com, leandro.tools@gmail.com, \\ ${ }^{2}$ Departamento de Engenharia Mecânica, Universidade Federal do Paraná, Curitiba, Paraná, Brasil, sofmat@ufpr.br
}

\begin{abstract}
Resumo
A manufatura aditiva (MA) é uma evolução da prototipagem rápida no sentido que cria um produto físico a partir de um arquivo digital (CAD 3D), entretanto sua utilização extrapola a produção de protótipos. Esta técnica envolve o projeto de um componente em camadas, estas multicamadas são depositadas por processos de soldagem produzindo o componente sem a necessidade de moldes ou outras ferramentas. Inicialmente esta tecnologia quase se restringia a processos de alta densidade de energia entretanto a busca por maior competitividade levou utilização de processos de soldagem a arco, utilizando material de adição na forma de arame ou de pó. Este trabalho tem por objetivo revisar as informações mais recentes com particular destaque para a MA utilizando processos a arco. São destacados os desafios envolvidos na fabricação de componentes por MA tanto na deposição das multicamadas como na fase de acabamento.
\end{abstract}

Palavras-chave: Manufatura aditiva, Prototipagem Rápida, deposição de multicamadas, processos a arco

\section{Abstract}

Additive manufacturing (AM) can be considered an evolution from Rapid prototyping as it allows to manufacture a component from a computer file (CAD 3D) though its applications extrapolate the production of prototypes. This technique involves the layered design of a component and subsequent welding deposition of the multilayer structure to produce parts without the need for molds or other tools. Although AM is frequently associated with the use of high density processes the need for higher competitiveness expanded its range of technologies to include arc welding processes. This paper aims to summarize up-to-date information on AM, particularly involving arc welding processes. Emphasis is given on the challenges associated with the building-up of components during multilayered deposition and on post-deposition procedures.

Key-words:Additive manufacturing rapid prototyping, multilayer deposition, arc processes

\section{Introdução}

A "Manufatura Aditiva" - MA (Additive Manufacturing$A M$ ) é definida como um grupo de tecnologias que utiliza uma abordagem camada por camada para criar objetos com forma livre; da base ao topo. A MA consiste em converter um modelo CAD 3D, Fig. 1a, em camadas, Fig. 1b, e a partir dessa informação determinar a trajetória (linguagem CNC) e os parâmetros de deposição, Fig. 1c, que posteriormente são processados por quatro componentes básicos: controlador CNC; sistema de movimentação; fonte de energia; e um sistema alimentação do material de adição (GIBSON et al., 2010).

$\mathrm{O}$ termo prototipagem rápida ( $\mathrm{RP}$ - Rapid Prototyping), no

Recebido em 09/07/2014, texto final em 09/07/2014.

DOI: 10.1590/0104-9224/SI1902.11 contexto do desenvolvimento de produto, foi extensivamente usado para descrever tecnologias que criam produtos físicos, diretamente, a partir de um arquivo digital (CAD 3D). Atualmente essas tecnologias extrapolam a prototipagem, sendo possível a fabricação de componentes funcionais, diretamente, a partir de um arquivo digital. Diante disso, um comitê técnico da ASTM concordou que uma nova terminologia deveria ser adotada e chegaram ao consenso de que o termo "Manufatura Aditiva" representa melhor esse grupo de tecnologias. (GIBSON et al., 2010).

Todos os equipamentos de MA comercializados atualmente são baseados da abordagem "camada por camada" e o diferencial entre estes equipamentos está no material que pode ser processado. A Tab. 1 resume os processos de manufatura aditiva em função dos materiais possíveis de processar. Essa habilidade de processar diferentes materiais nucleou uma nova abordagem denominada Manufatura Aditiva com Múltiplos Materiais - MAMM ( ou MMAM -Multiple Materials Additive Manufacturing) como citado por GIBSON et al., 2010. 


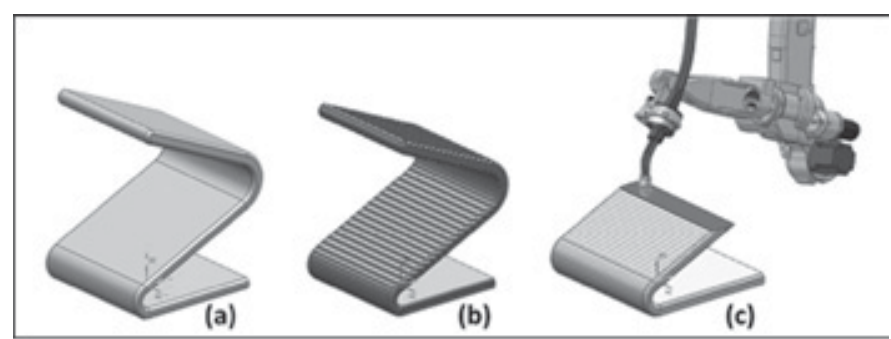

Figura 1. Esquema da AM: (a) modelo CAD 3D; (b) divisão do modelo em camadas; (c) deposição das camadas (Fonte: Os autores)

Tabela 1. Processos de manufatura aditiva (GIBSON et al., 2010)

\begin{tabular}{|c|c|c|c|}
\hline Processo & Descrição & $\begin{array}{c}\text { Metodo de } \\
\text { AM }\end{array}$ & Material \\
\hline Fotopolimerização & $\begin{array}{l}\text { Um polímero } \\
\text { fotocurável } \\
\text { é curado } \\
\text { seletivamente } \\
\text { usando uma } \\
\text { fonte de luz }\end{array}$ & Laser & $\begin{array}{l}\text { Polímero } \\
\text { fotocurável }\end{array}$ \\
\hline $\begin{array}{l}\text { Modelagem por } \\
\text { extrusão }\end{array}$ & $\begin{array}{l}\text { Material é } \\
\text { depositado de } \\
\text { forma seletiva } \\
\text { através de } \\
\text { um cabeçote } \\
\text { extrusor }\end{array}$ & $\begin{array}{l}\text { Aquecimento } \\
\text { por uma } \\
\text { resistência } \\
\text { elétrica }\end{array}$ & $\begin{array}{c}\text { Polímeros, } \\
\text { cerâmicas e } \\
\text { metais }\end{array}$ \\
\hline $\begin{array}{l}\text { Fusão de pós pré- } \\
\text { depositados }\end{array}$ & $\begin{array}{l}\text { Um feixe } \\
\text { eletrônico funde } \\
\text { seletivamente } \\
\text { regiões de um } \\
\text { leito com pó } \\
\text { pré-depositado }\end{array}$ & $\begin{array}{l}\text { Laser e feixe } \\
\text { de elétrons }\end{array}$ & $\begin{array}{l}\text { Polímeros, } \\
\text { cerâmicas e } \\
\text { metais }\end{array}$ \\
\hline Cladding 3D & $\begin{array}{l}\text { O material } \\
\text { de adição na } \\
\text { forma de pós } \\
\text { é injetado } \\
\text { diretamente no } \\
\text { feixe/poça }\end{array}$ & Laser e PTA & $\begin{array}{l}\text { Metais e } \\
\text { cerâmicas }\end{array}$ \\
\hline Arco elétrico & $\begin{array}{l}\text { Fonte de energia } \\
\text { é um arco } \\
\text { elétrico que } \\
\text { funde o material } \\
\text { de adição } \\
\text { (pó/arame) }\end{array}$ & $\begin{array}{l}\text { PTA, Plasma } \\
\text { arame, TIG, } \\
\text { MIG/MAG }\end{array}$ & Metais \\
\hline
\end{tabular}

A MAMM permite melhorar o desempenho de um componente através do design ou arquitetura microestrutural, ou seja, utilizando diferentes materiais/composições químicas em regiões estratégicas de um componente. A relevância deste procedimento é destacada por Murr et al. (2012a), que explicam que o controle da arquitetura microestrutural pode alterar/ estender o tradicional paradigma da ciência e engenharia dos materiais, do tripé estrutura-propriedade-processamento e seu impacto no desempenho (pirâmide de base triangular), para os quatro pilares da arquitetura microestrutural (pirâmide de base quadrangular), Fig. 2.

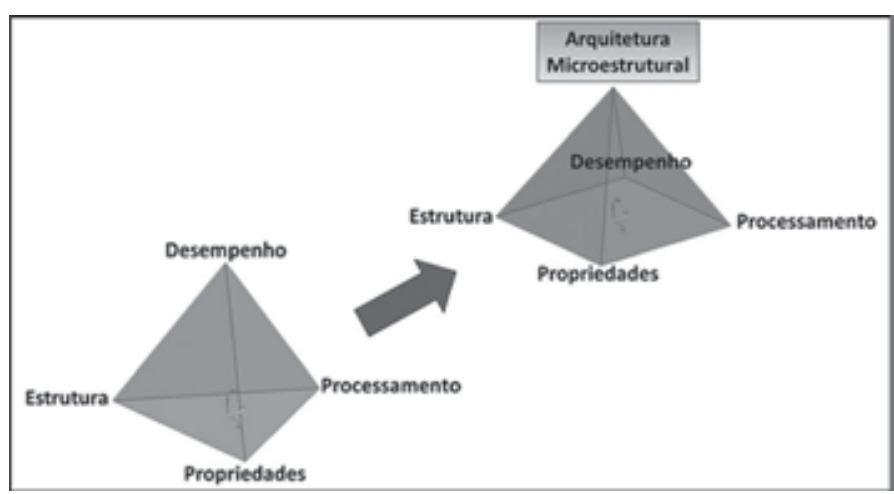

Figura 2. Impacto da MMAM na arquitetura microestrutural, adaptação de Murr et al. (2012a)

Muitas aplicações podem, potencialmente, ser beneficiadas com o desenvolvimento da MMAM, que permite adequar propriedades específicas nas regiões mais solicitadas do componente. Por exemplo, condutividade térmica nos canais de refrigeração num molde de injeção de alumínio sobre pressão, ou resistência à erosão, corrosão e à alta temperatura nas partes quentes de turbinas aeroderivativas, destacando-se ainda a redução com relação às perdas no processo de fabricação de componentes da indústria aeroespacial, etc.

Apesar das grandes vantagens, os processos de manufatura aditiva possuem diversos obstáculos, os quais necessitam ser superados a fim de tornar esses processos mais viáveis. Entre estes pode-se citar o tempo de fabricação de um único componente, que é muito maior comparado ao dos processos de fabricação em massa, disponibilidade de máquinas com a flexibilidade necessária para trabalhar com diversos materiais, geometrias e precisão adequada, que variam de acordo com a peça e custo de equipamentos (BOURELL et al., 2009). Porém, com a maior utilização e disponibilização dos resultados de pesquisa relacionados a essa área de manufatura, aumenta a competitividade desta técnica. Um exemplo são as próprias impressoras 3D de polímeros, que atualmente já possuem um custo mais acessível possibilitando novas possibilidades de negócios e investimentos na área da manufatura aditiva.

Entretanto, a manufatura aditiva - MA ou a MAMM utilizando ligas metálicas ainda se restringe a poucos componentes. Considerando as características atrativas das técnicas de manufatura aditiva e o importante papel dos processos de soldagem e da metalurgia da soldagem, é apresentado neste trabalho destaques da MA com metais, focando especificamente nos processos que utilizam o laser e o arco elétrico como fonte de energia, bem como características das ligas mais utilizadas.

\section{Seleção do processo e material}

A deposição de metais na tecnologia de manufatura aditiva pode ocorrer através de diferentes processos de soldagem a arco (Plasma, MIG e TIG), Laser cladding, Selective Laser Melting, 
Electro Beam Melting, sinterização de pós e por variáveis desses mesmos processos.

A seleção do processo a ser utilizado depende fortemente da geometria a ser fabricada. Peças pequenas, com design complexo, necessitam de baixas taxas de deposição, e desta forma os processos a laser, feixe de elétrons e micro-plasma são os mais indicados. O processamento de componentes com maiores dimensões utiliza processos com maiores taxas de deposição, por exemplo, os de soldagem a arco. A Fig. 3 apresenta uma comparação das larguras de cordões únicos sobrepostos para construção de uma parede fina, possíveis de obter por diferentes processos. As informações aqui apresentadas são baseados na literatura recente podendo ser passível de atualização com a disponibilidade de informações relacionada à MA.

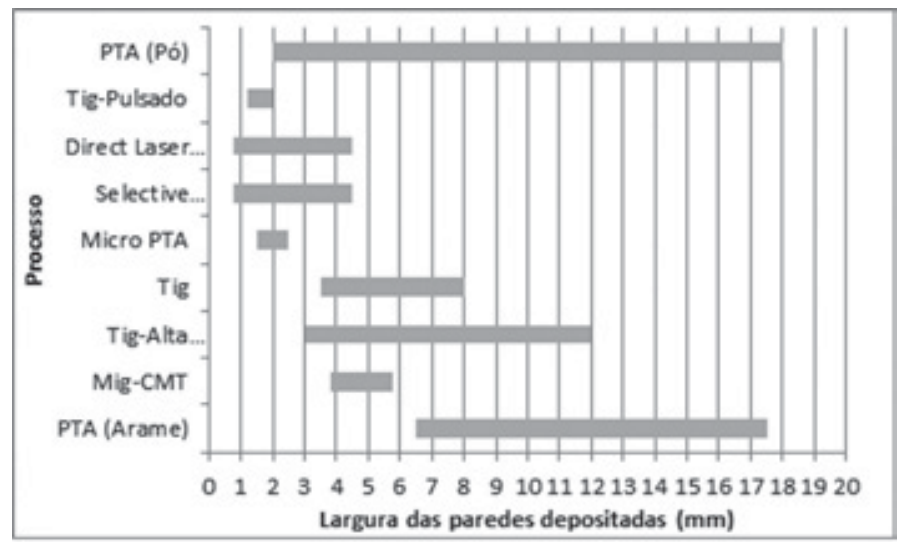

Figura 3. Largura de cordões únicos depositados por diferentes processos (JHAVAR et al., 2014; MARTINA et al., 2012)

A gama de metais já aplicada pelas técnicas de manufatura aditiva, segundo a European Powder Metallurgy Association, inclui ligas de titânio, alumínio, níquel, cobalto, aços (inoxidável, ferramentas e baixa liga) e metais preciosos. Estas ligas são utilizadas na forma de arame ou pó de acordo com o processo utilizado.

A seleção do material a ser utilizado e do processo estão fortemente interligadas. Determinadas ligas apresentam maior ou menor facilidade de processamento, dependendo do processo utilizado. Por exemplo, superligas de níquel são propícias à formação de trincas a quente, dificultando o uso de processos que produzem grande aquecimento na peça (BIN e GASSER, 2011). Grande parte das informações necessárias para a seleção desses processos são baseadas em conhecimentos adquiridos em procedimentos de soldagem, pois pesquisas exclusivas de manufatura aditiva para muitas combinações de ligas e processos ainda são escassas.

\subsection{Deposições a laser e feixe de elétrons}

Os sistemas de manufatura aditiva mais utilizados são aqueles assistidos por feixes de laser ou de elétrons, como comenta GIBSON et al. 2012. A justificativa para ampla utilização está na grande velocidade e precisão de deposição ou fusão dos feixes, somado a elevada densidade de energia que faz com a cada passe apenas um pequeno volume do "substrato" seja fundido, além das rápidas velocidades de resfriamentos decorrentes dos grandes gradientes de temperatura impostos (STENN et al. 2010).

Existem algumas variações para este grupo de tecnologias com alta densidade de energia, particularmente aquelas assistidas por laser. Por exemplo, Murr e colaboradores (MURR et. al, 2012a, 2012b e 2012c) realizaram estudos utilizando uma mesa de fusão, com um sistema SLM (Selective Laser Melting). Este sistema consiste na deposição de uma camada da liga em pó sobre a mesa de fusão e o feixe de laser segue uma trajetória pré-definida, fundindo de maneira seletiva, os pósmetálicos atomizados pré-depositados. A cada ciclo uma nova camada de pós é pré-depositada e à medida que o componente cresce verticalmente, a mesa de fusão desce, mantendo a mesma distância focal. Quando o componente está formado é retirado o pó excedente e, se necessário, a peça segue para acabamento final por usinagem.

Outra abordagem foi adotada por Brandl e colaboradores (BRANDL et al., 2011a e 2011b, 2011c, 2012d e 2012e), e em seguida por outros grupos de pesquisa, que utilizam um sistema a laser alimentado com arame frio. Brandl et al. explicam que a alimentação com arame ganhou destaque devido a maior produtividade e eficiência, quando comparado com sistemas que operam com pós pré-depositados.

Liu et al, 2014, concordam com Brandl e colaboradores em relação a maior produção, mas explicam que a baixa absorção de energia pelo arame frio é a principal limitação desta abordagem e sugere a alimentação com arame quente como uma solução efetiva para aumentar a produtividade. Esta preocupação não é tão recente, como mostraram Nurminen et al. (2006) em trabalho que comparou três sistemas assistidos por laser, alimentados por pó, arame frio e arame quente. Concluíram que a taxa de deposição, do sistema com arame quente é quatro vezes maior do que a dos outros sistemas.

Apesar da maior taxa de alimentação dos sistemas assistidos por laser alimentados com arame quente, é importante destacar que a composição química, nesta abordagem, é limitada à composição do arame. Característica que contrasta com os sistemas com alimentação com pó, que permitem que a composição possa ser facilmente variada, além de possibilitarem a produção de ligas in-situ e a variação da composição química/propriedades em regiões estratégicas do componente, procedimento que Murr, et. al 2012a denominaram de arquitetura microestrutural.

O cladding 3D é outra variação da MA assistida por laser. Nesta configuração o pó é alimentado diretamente no feixe/poça e pode ser utilizada tanto para fabricação quanto para reparo de componentes desgastados. Bin e Gasser (2011), utilizando superligas com baixa soldabilidade e adotando este sistema este sistema conseguiram reparar componentes de turbinas aeroderivativas com sucesso.

Bi, Sun e Gasser (2013), explicam que o cladding 3D tem como diferencial, frente aos processos convencionais utilizados em reparos (PTA e TIG), o melhor controle dos parâmetros. Variando-se a potência do laser a temperatura de trabalho que a área do componente está exposta é controlada, o que 
permite evitar defeitos de soldagem como trincas e minimizar a degradação metalúrgica do material depositado em passes anteriores.

A recuperação de geometrias complexas é uma extensão da MA, que tem grande potencial tecnológico e interesse industrial, principalmente em se tratando de componentes de alto valor agregado. Neste processo de recuperação, a sequência de etapas envolvidas é um pouco mais complexa, iniciando-se pelo levantamento da geometria original do componente desgastado (requer um modelo 3D), passando pela avaliação de eventuais deformações devido ao aquecimento da peça durante o processo de recuperação/reconstrução, avaliação da integridade e propriedades do componente recuperado e terminando com o pós-processamento, ou seja, usinagem via CAM (YILMAZ et. al 2010).

\subsection{Deposição com soldagem a arco}

A utilização de processos de alta densidade de energia na manufatura aditiva apresenta vantagens competitivas, mas possuem uma baixa eficiência energética (JHAVAR et al., 2014), motivando diversas pesquisas que buscam processos mais eficazes. Processos com arco elétrico se enquadram nesse quesito por possuir maior eficiência energética e exibindo uma ampla abrangência em relação à quantidade de material depositado, possibilitando a produção de peças maiores com maior rapidez. Deposições com o processo laser e feixe de elétrons possuem uma taxa de deposição entre 2 e $10 \mathrm{~g} / \mathrm{min}$, já processos a arco podem atingir valores superiores a $130 \mathrm{~g} / \mathrm{min}$ (SURYAKUMAR et al., 2011). Esse tema despertou a atenção de diversos grupos de pesquisa no mundo, podendo-se mencionar alguns deles nos EUA (Southern Methodist University, University of Michigan e University of Kentucky), Reino Unido (Cranfield University e Loughborough University), Alemanha (Fraunhofer Institutes), Coreia do Sul (KoreanInstitute of Science \& Technology), Japão (Joining and Welding Research Institute), India (Indian Institute of Technology) e Bélgica (Katholieke Universiteit Leuven).

As ligas utilizadas em deposições com arco elétrico são similares às utilizadas com o processo de laser, sendo possível encontrar pesquisas que focam em aços, alumínio e níquel. Mas a grande maioria das pesquisas estuda ligas de titânio, principalmente a liga Ti-6Al-4V (SKIBA et al., 2009). Essa liga tem aplicações no mercado aeronáutico e biomédico, que têm apoiado um grande número de pesquisas, buscando mapear a resposta dessa liga com diferentes processos de deposição. Um centro de pesquisa de referência nessa área é a Cranfield University, no Reino Unido, que já avaliou o efeito de diferentes processos e suas variações, como GTAW, CMT - Cold Metal Transfer - (ALMEIDA; WILLIAMS, 2010) e PlasmaArame (MARTINA et al., 2012). O foco destes trabalhos é o mapeamento de parâmetros de deposição, que permitam a construção de paredes finas, tendo sido demonstrado que o processo plasma permite deposições de paredes com maior escala e taxas de deposição, apesar do processo CMT permitir cordões individuais com maiores alturas.

Outro conjunto de ligas com grande potencial de aplicação na manufatura aditiva é o das ligas a base de níquel. As superligas de níquel tem aplicação no setor de energia e aeronáutica em consequência das excelentes propriedades em elevadas temperaturas (HENDERSON et al., 2004). A aplicação dessas ligas com processos à arco ocorre em casos onde há necessidade de altas taxas de deposição, tanto para trabalhos de construção como para reparos. Processos de soldagem GMAW, por exemplo, permitem depositar cordões individuais com larguras superiores a $12 \mathrm{~mm}$ e alturas de $2 \mathrm{~mm}$. Clark et al. (2008), utilizando a liga de níquel 718 na deposição de múltiplas camadas conseguiram obter paredes com larguras maiores que $30 \mathrm{~mm}$ e alturas superiores a $60 \mathrm{~mm}$, na construção de câmaras de combustão. Deposições com plasma também são bastante atrativas para processar ligas a base de níquel. Como esse processo promove um menor efeito térmico, a probabilidade de trincas nas ligas de níquel é menor do que nos outros processos a arco, além de que o custo de equipamento e processamento é significativamente menor do que dos processos de feixe de elétrons e laser (SU et al., 1997). Pesquisas demonstram que deposições utilizando a técnica de PTA apresentam a possibilidade de depositar esta liga com uma larga faixa de espessuras, com cordões variando de $2 \mathrm{~mm}$ até $10 \mathrm{~mm}$ de espessura. A Fig. 4 apresenta a imagem de uma deposição de múltiplas camadas da liga Inconel 625 processada por PTA.

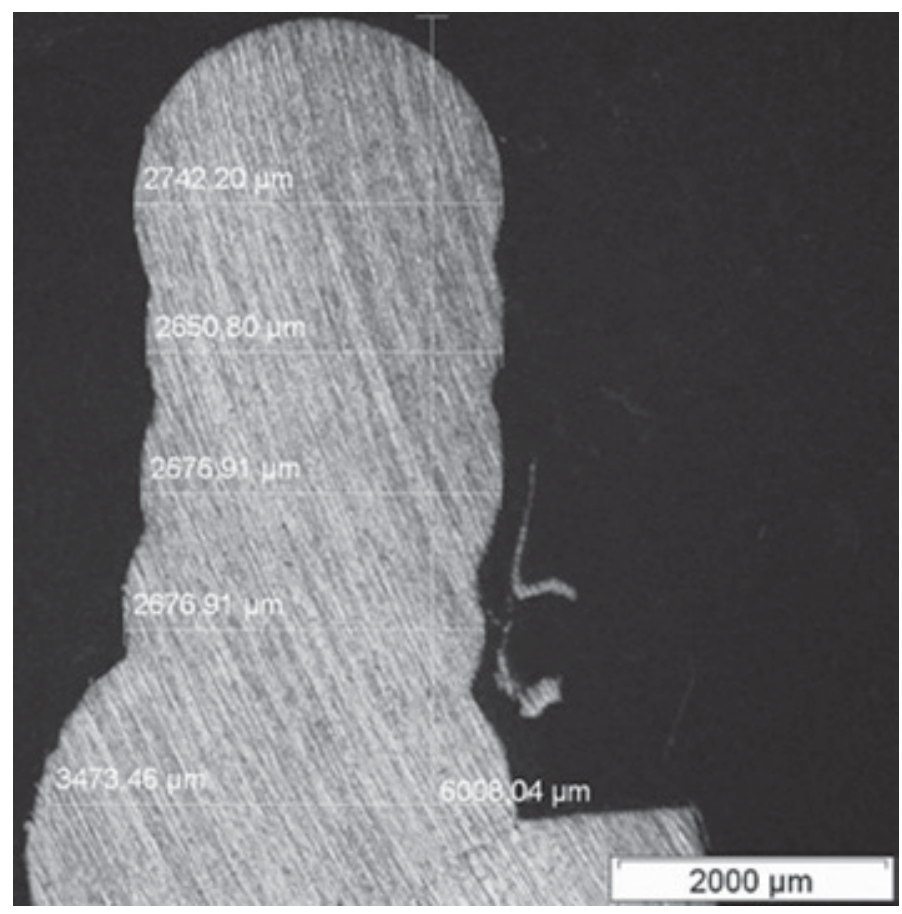

Figura 4. Deposição de múltiplos cordões da liga Inconel 625 com processo plasma por arco transferido (Fonte: os autores)

Um tipo de material que tende a aumentar as aplicações relacionadas à manufatura aditiva são os intermetálicos. Essas ligas possuem ótimas propriedades para trabalhos em altas pressões e/ou elevadas temperaturas, como mencionado por D’Oliveira et al. (2011), sendo mais frequentemente utilizadas como ligas estruturais de alta resistência mecânica. Entretanto, o alto custo e as dificuldades de processamento são desafios a serem vencidos, como por exemplo, o da ocorrência de 


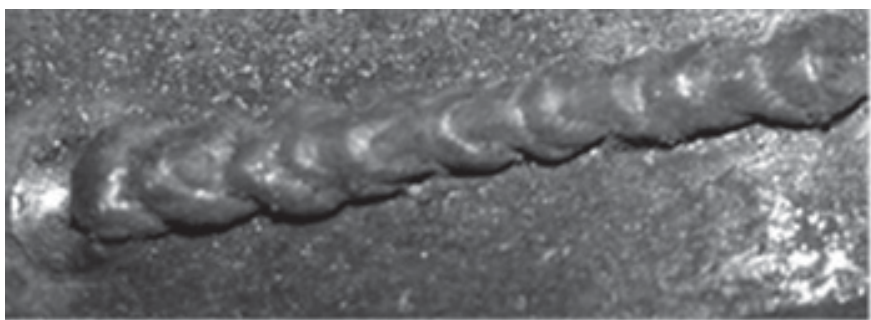

(a)

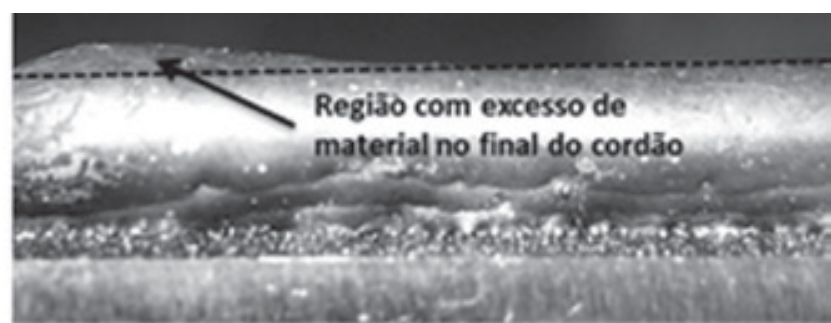

(b)

Figura 5. Defeitos de morfologia observados em cordões depositados para manufatura aditiva; (a) cordão não homogêneo; (b) cordão com excesso de deposição na extremidade (Fonte: os autores)

uma reação exotérmica durante síntese, o que pode modificar características importantes do processo, como temperatura (KATOU et al., 2007). Apesar do maior controle necessário quando se processam ligas intermetálicas, a deposição de NiAl, Ti-Ni e Ti-Fe com o processo GTAW, visando a fabricação "free form", obteve sucesso em produzir cordões com larguras reduzidas, próximas a $1 \mathrm{~mm}$, livres de trincas e poros e com composição uniforme (KATOU et al., 2007). Trabalhos com PTA utilizando misturas de pós de $\mathrm{Ni}$ e $\mathrm{Al}$, permitiram obter cordões de aluminetos processados in-situ, isto é durante a deposição, com espessuras acima de $3 \mathrm{~mm}$, e com estrutura e composições homogêneas (D'OLIVEIRA et al., 2011). Estas ligas são particularmente interessantes para serem aplicadas em conjunto com ligas metálicas para aplicações que possam usufruir de componentes processados por MAMM - Manufatura Aditiva com Multíplos Materiais.

$\mathrm{O}$ reparo de componentes com geometria complexa e alto custo de fabricação é um nicho com grande potencial de aplicação para a MA. Um exemplo são moldes utilizados em processos de conformação, extrusão e outros. Esses componentes são fabricados por ligas de alta resistência mecânica, em geral, aços ferramentas, e sofrem desgaste pelo uso. O reparo desses componentes em muitos casos é inviável devido a sua geometria complexa e/ou ao elevado custo. A manufatura aditiva tornase um processo competitivo nesse mercado, como mostram trabalhos com micro-plasma, que depositaram o aço AISI P20 (aço com cromo e molibdênio), criando paredes finas com a sobredeposição de cordões (JHAVAR et al., 2014). Essas deposições resultaram em cordões com $2 \mathrm{~mm}$ de espessura, homogêneos e livres de poros ou trincas, possibilitando a reconstrução de geometrias complexas. Além de aços ferramentas, também são encontrados trabalhos com aços inoxidáveis visando componentes com resistência à corrosão e alta ductilidade. A sobreposição de cordões de aço inoxidável AISI 308 realizadas por GTAW, permitiu construir paredes com $8 \mathrm{~mm}$ de espessura e mais de $30 \mathrm{~mm}$ de altura, sem a presença de poros ou trincas, mantendo-se constantes as propriedades mecânicas do material constante por toda a deposição, e com valores similares a de outros métodos de fabricação de peças (SKIBA et al., 2009).

\section{Controle de processamento na construção do compo- nente}

A construção de um componente por MA apresenta diversos momentos determinantes para o sucesso do procedimento. A deposição do primeiro cordão está entre os fatores que requerem cuidadosa análise. Nesse primeiro cordão, em reparos, procurase uma boa ligação metalúrgica com o substrato, além de uma espessura e morfologia adequadas. Já nos testes para a formação de uma "parede" pela sobredeposição de múltiplas camadas é analisada a morfologia e microestrutura dos depósitos. O controle da morfologia visa garantir a continuidade dos cordões, variação de altura ou defeitos. A continuidade dos cordões está associada à seleção dos parâmetros de processamento, sendo que uma seleção inadequada pode resultar em cordões com grande variação de largura ou falta de fusão do metal depositado (MARTINA et al., 2012). A Fig. 5a apresenta exemplo de um cordão pouco homogêneo. As variações de altura de cordões relacionam-se a mudanças nas condições de processamento em pontos específicos, como eventuais variações de espessura do substrato que podem resultar no aumento da temperatura na deposição ou mudança da velocidade de deposição, o que é comum nas extremidades dos cordões (BIN; GASSER, 2011). A Fig. 5b apresenta um exemplo de variação na altura na extremidade do cordão, ocorrida pela parada da tocha nesse local, aumentando a energia imposta nessa região. Outros defeitos que também podem ser observados nos cordões depositados são grandes poros, trincas e oxidação, que podem comprometer as propriedades do componente construído ou reparado pela MA.

A geometria dos cordões é uma característica de grande importância na manufatura aditiva. É a base para programar o processo de fabricação, como a quantidade de passes e posição de cada cordão. Com isso, as pesquisas buscam cordões com espessuras que permitam reduzir o número de passes necessário para se obter o formato final, diminuindo, assim, custos de deposição e de usinagem. Um exemplo onde a geometria dos cordões é de grande importância é a restauração de componentes de turbinas a gás, no qual paredes finas de aproximadamente $1 \mathrm{~mm}$ devem ser recuperadas após desgaste durante operação. Nesses casos, a reconstrução requer que se depositem cordões com espessura pouco superior, permitindo a remoção de material após usinagem para dar o acabamento adequado à peça (BIN e GASSER, 2011). Para uma análise da real geometria possível de se construir após a deposição de múltiplas camadas, uma ferramenta utilizada é a medição das espessuras total e efetiva da "parede" depositada, assim como a altura dos cordões (MARTINA et al., 2012). A espessura total 
é a maior espessura obtida nos cordões depositados durante o processamento, enquanto a espessura efetiva e a maior espessura possível de obter uma parede fina, após usinagem. A Fig. 6 apresenta um esquema das medições das espessuras efetiva e total. A altura medida nessa análise é a altura individual de cada cordão, que junto com os valores de espessura total e efetiva, permitem a programação da deposição para a construção final do componente.
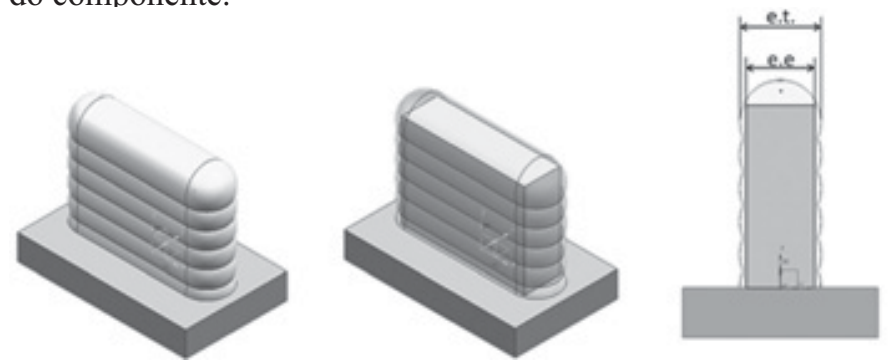

Figura 6. Esquema de medição das espessuras total (e.t.) e efetiva (e.e.) em deposição de manufatura aditiva (Fonte: os autores)

Além das características já mencionadas, vários outros fatores devem ser levados em conta durante o processo da manufatura aditiva, buscando a produção de componentes íntegros. Entre esses, pode-se mencionar o ciclo térmico associado às sobredeposições de cordões, a trajetória de deposição, a temperatura entre passes, a variação da distância entre tocha e peça, a distância entre deposição de camadas laterais, etc.

O ciclo térmico gerado durante deposição é de grande importância nos processos de manufatura aditiva, uma vez que o componente sofre múltiplas exposições à temperatura devido à deposição de múltiplas camadas. Esta condição gera uma complexa distribuição do gradiente de temperatura pela peça, o que influencia a distribuição de tensões, deformações, microestrutura e, consequentemente, o desempenho da peça produzida (ZHAO et al., 2011). Em posições como início e fim do cordão há uma variação maior, já na parte central as tensões tende a se manter estáveis (ZHAO et al., 2012).

A trajetória de deposição também influencia as características finais da peça. As duas principais táticas para construção de paredes finas são a direção igual, fig. 7a, e a direção reversa, Fig. 7b. A variação dessa trajetória influencia na estrutura solidificada, no gradiente térmico e nas tensões geradas pela deposição. A estrutura gerada nas deposições com direção igual tende ser mais homogênea, com uma direção de solidificação constante em todas as camadas, enquanto nas deposições com direção reversa, a direção de crescimento, que segue o escoamento de calor, muda a cada camada, interrompendo o crescimento das dendritas (DINDA et al., 2009). As tensões geradas durante a deposição pelo gradiente térmico também variam dependendo da trajetória utilizada, sendo que com a mudança de direção entre os cordões, as tensões ao longo da deposição tende a ser menor (ZHAO et al., 2012).

A variação de distância entre tocha e a peça também influencia nas características finais da peça, ao determinar o calor fornecido ao material pela fonte de energia, diminuindo a área afetada pela temperatura e quantidade de material depositado, o que interfere diretamente na geometria e gradientes térmicos dos cordões depositados. Portando, a necessidade de manter um distância tocha-peça constante possuí grande importância, inclusive motivando pesquisas para desenvolvimento de técnicas para garantir tal condição (XIONG; ZHANG, 2014).
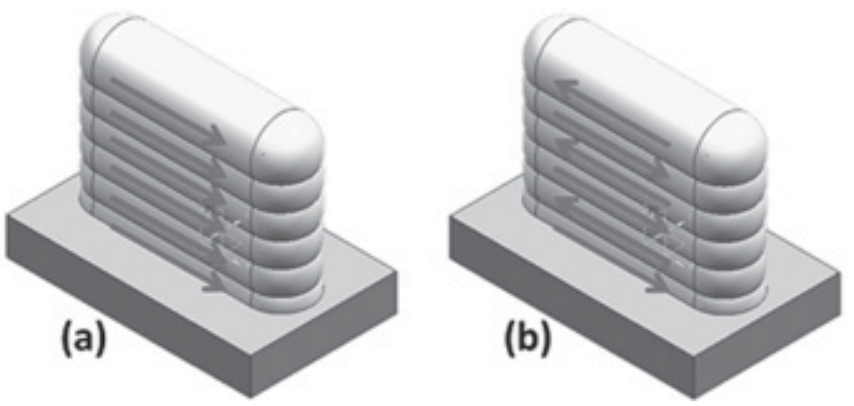

Figura 7. Duas abordagens (tácticas) de direção de deposição: (a) direção igual; (b) direção reversa (Fonte: Os autores)

A temperatura entre passes é outro fator de grande importância e que depende da liga depositada. Ligas à base de níquel, por exemplo, podem apresentar trincas de reaquecimento devido aos ciclos térmicos impostos durante o processo

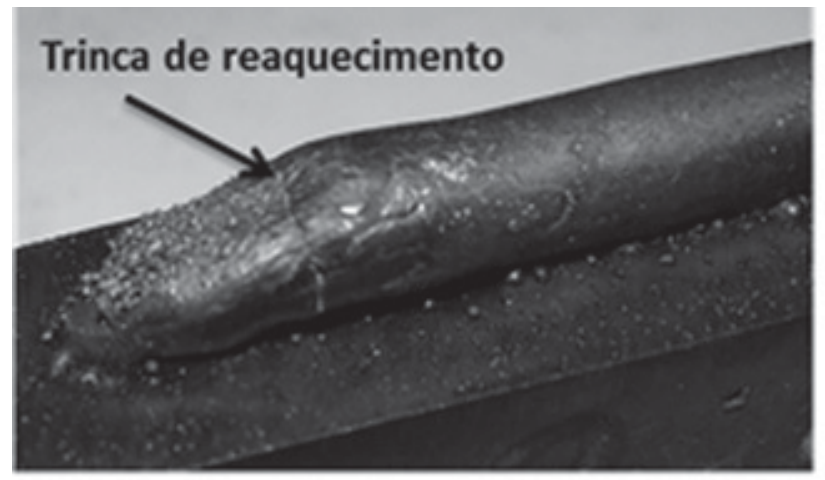

(a)

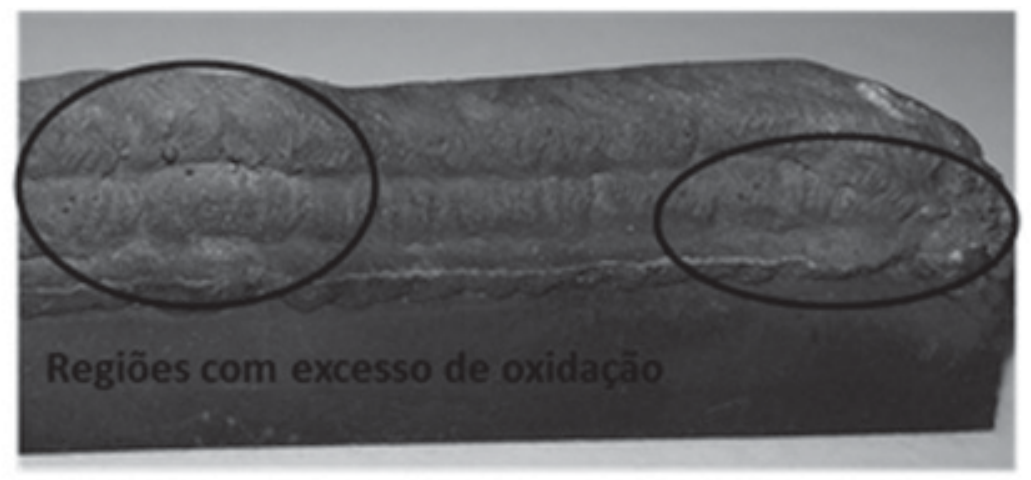

(b)

Figura 8. Deposições de níquel com problemas (a) de trinca por reaquecimento e (b) oxidação na deposição de multicamadas sem atmosfera protetora ( Fonte: Os autores) 
(HENDERSON et al., 2004), Fig. 8a. Nesses casos, realizar a deposição mantendo uma temperatura de pré-aquecimento a cada deposição de camada, diminui o gradiente térmico gerado no processo, consequentemente diminuindo a possibilidade de trincas (FLOWERS et al., 2000).

Outra questão que deve ser analisada no processo é a necessidade da utilização de atmosfera protetora durante a "construção" da parede. Diversas ligas apresentam oxidação nas temperaturas de processamento, prejudicando a qualidade das propriedades mecânicas das deposições. Exemplos de materiais que necessitam desse cuidado são superligas de níquel e ligas a base de titânio (HENDERSON et al., 2004; MARTINA et al., 2012), Fig. 8b.

\section{Pós-processamento}

Para a manufatura de um componente por MA é necessário realizar um processo de acabamento após deposição, buscando as dimensões finais requeridas, recorrendo-se para isso a processos de usinagem. Além disso, para a maioria dos materiais depositados é preciso buscar uma homogeneidade na microestrutura e propriedades mecânicas dos cordões depositados.

Amicroestrutura dos componentes fabricados pelamanufatura aditiva é de grande complexidade, e depende fortemente do processo de deposição e dos parâmetros selecionados para construção do componente. As características do processo de deposição, como parâmetros de soldagem, direção de deposição, temperatura de pré-aquecimento e tempo entre passes, têm influência direta nas condições de solidificação, como tempo de solidificação e direção de escoamento de calor. O tempo de solidificação afeta de forma significativa as ligas endurecidas por precipitação, tanto na saturação das soluções sólidas como na quantidade, distribuição e morfologia dos precipitados que compõem a estrutura formada, assim como na formação de eventuais fases metaestáveis. Já, a direção de resfriamento pode determinar a direção do crescimento das dendritas e precipitados (FRAZIER, 2014).

Os gradientes térmicos impostos a cada deposição são também responsáveis por variações no refino da estrutura ao longo da altura da parede construída. A Fig. 9 apresenta um exemplo de gradiente imposto a cada deposição durante um processo de MA, observando-se sua variação com o crescimento do componente. A modificação da microestrutura em cada cordão, ocorrida pela repetição dos gradientes térmicos, é discutida em diversos trabalhos para diferentes materiais. Em ligas de titânio, como a Ti-6Al-4V, por exemplo, é observada a formação de grãos epitaxiais, compostos pela fase $\beta$, em crescimento junto ao substrato. Ainda no primeiro cordão, é observada uma fina estrutura de grãos equiaxiais, devido a maior eficiência de extração de calor no primeiro cordão pela proximidade com o substrato. Já nos cordões superiores, há menos grãos $\beta$ nucleando, porém com maiores dimensões (ALMEIDA et al., 2010). Em superligas de níquel também ocorrem variações no refino dos grãos, assim como a direção de crescimento deles. Foi observada a formação de grãos epitaxiais próximos ao substrato sendo que em cordões intermediários, há presença de crescimento celular e em cordões superiores evidências de crescimento equiaxial (BI et al., 2011 e 2013).

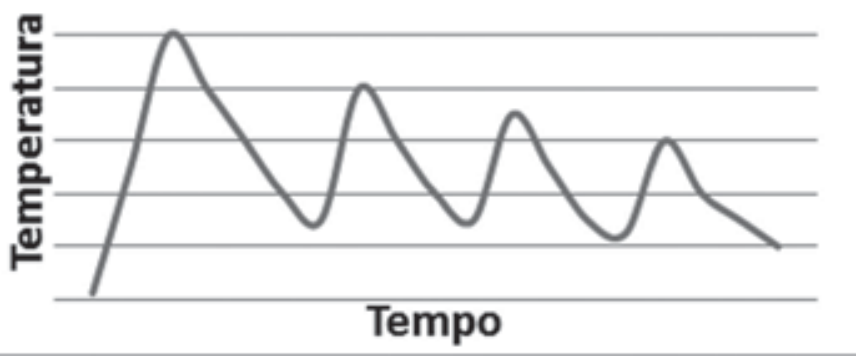

Figura 9. Exemplo de gradiente térmico gerado em um cordão durante processo de manufatura aditiva (Fonte: Os autores)

O impactos dos parâmetros de deposição na variação de estrutura formada na sobredeposição de cordões está apresentado na fig. 10. Destaca-se a continuidade da estrutura entre os diferentes cordões, e como a deposição de diferentes camadas afeta o crescimento das dendritas, com impactos na uniformidade das propriedades mecânicas da parede depositada. Medições de microdureza, Fig. 11, confirmam a variação de dureza à medida que se constrói o componente, sendo possível identificar o impacto da sobreposição dos cordões ao se correlacionar o perfil de dureza após deposição de 1, 3 e 5 cordões, confirmando assim a importância de se controlar os diferentes parâmetros de processamento.

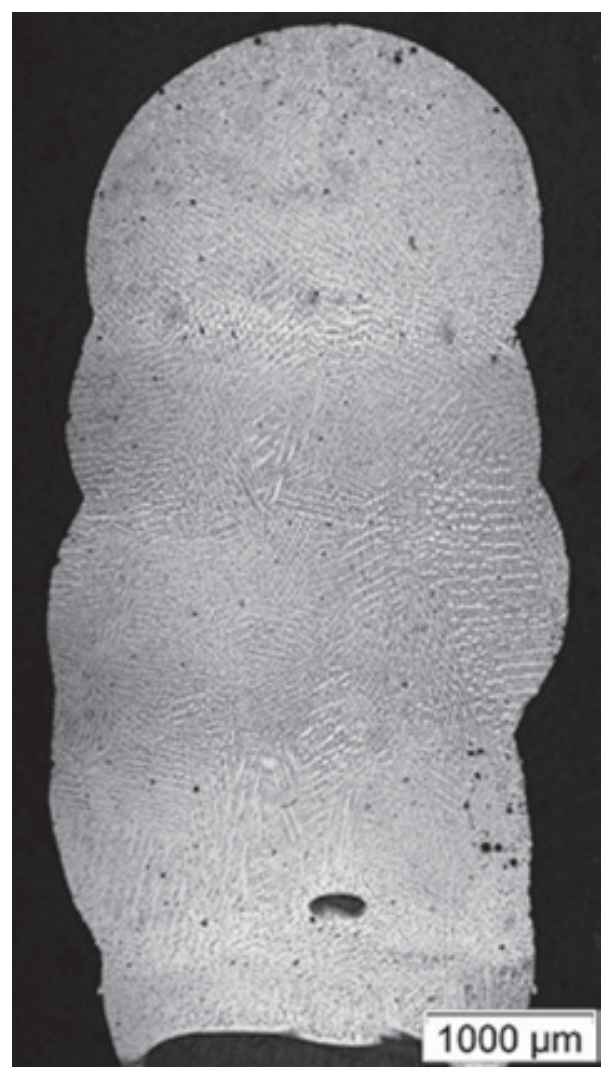

Figura 10. Seção transversal de uma deposição de multicamadas com superliga de níquel, apresentando variação na microestrutura formada ( Fonte: os Autores) 


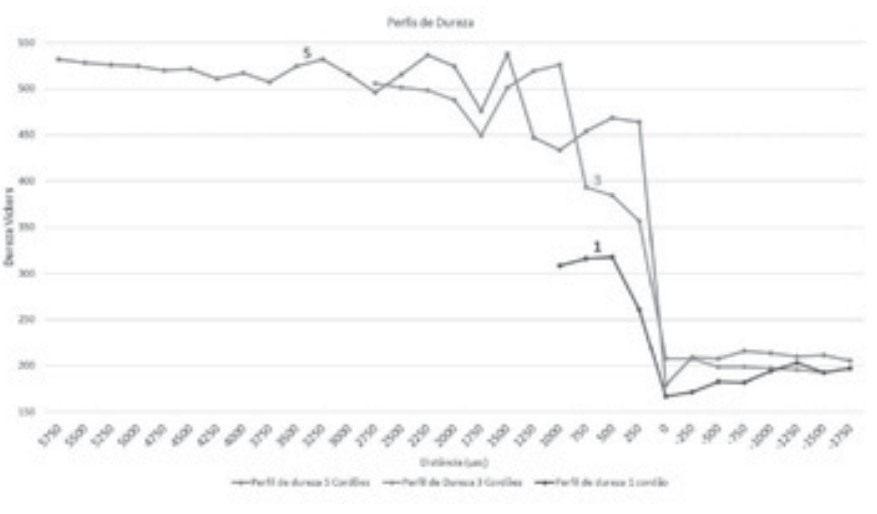

Figura 11. Variação da dureza em uma superliga de níquel influenciada pela deposição de multicamadas ( Fonte: os Autores)

Nestas situações há a necessidade de tratamentos térmicos para homogeneização da estrutura depositada. Esses tratamentos devem ser projetados considerando-se informações obtidas dos diagramas de fases das ligas depositadas, uma vez que, além do alivio de tensões e maior uniformidade da estrutura, etapas de solubilização e precipitação têm de ser conduzidas em temperaturas que dependem de cada composição química. As superligas de níquel, por exemplo, são expostas a tratamentos para a homogeneização da estrutura de solidificação, como o apresentado por Dinda et al. (2009), no qual uma liga Inconel 625 após tratamento em temperatura de $1200^{\circ} \mathrm{C}$ apresentou uma estrutura de grãos equiaxiais, mas com distribuição não uniforme do precipitado $\gamma^{\prime}$. Sajjadi et al. (2006) sugerem uma otimização dos parâmetros de tratamento térmico de forma a garantir também um controle na distribuição de precipitados.

\section{Comentários Finais}

- A manufatura aditiva apresenta-se, no mercado global, com um grande potencial de utilização na área de manufatura de componentes de alta complexidade geométrica e de propriedades mecânicas. Atualmente esse processo é visto por fabricantes de diversos setores, como aeronáutico, de energia e biomedicina como uma revolução na fabricação de diversos componentes.

- A aplicação de processos de soldagem na manufatura aditiva faz com que os conhecimentos na área de soldagem sejam de grande importância no inicio do desenvolvimento dessa técnica de manufatura, permitindo assim uma evolução mais rápida do processo. Inicialmente técnicas de alta densidade de energia como o Laser Cladding e a deposição por feixe de elétrons eram as únicas encontradas no âmbito da manufatura aditiva. Porém, a utilização de processos a arco aparece como alternativa para aumentar a competitividade da MA. Utilizando materiais de alimentação na forma de pó ou arame também permitem maior flexibilidade na construção de componentes com gradiente de propriedades.

- Os procedimentos de MA não são uma mera extrapolação de procedimentos de soldagem, requerendo um rigoroso controle de parâmetros para a deposição sucessiva das múltiplas camadas. A determinação de uma sequencia correta de deposição garante o controle da geometria do componente e tem impacto em suas propriedades, sendo na maioria dos casos necessário um tratamento térmico para homogeneização da estrutura. Assim, sendo necessário um maior entendimento dos fenômenos envolvidos pela interação de diferentes materiais nos processos de manufatura aditiva, de forma a mapear as reais possibilidades desse processo.

\section{Referências Bibliográficas}

AKULA, S.; KARUNAKARAN, K.P. Hybrid adaptive layer manufacturing: An Intelligent art of direct metal rapid tooling process. Robotics and Computer-Integrated Manufacturing, p. 113-123, 2006.

ALMEIDA, P.M.S.; WILLIAMS, S.W. Innovative process model of Ti-6Al-4V additive layer manufacturing using cold metal transfer (CMT). In: 21st International Solid Freeform Fabrication Symposium, Austin, TX, August 9-11, 2010.

BI, G.; GASSER, A. Restoration of Nickel-Base Turbine Blade Knife-Edges with Controlled Laser Aided Additive Manufacturing. Physics Procedia, p. 402-409. mar. 2011.

BI, G.; SU, C. N.; GASSER, A. Study on influential factors for process monitoring and control in laser aided additive manufacturing. Journal Of Materials Processing Technology. p. 463-468. out. 2013.

BOURELL, D.L.; LEU, M.C.; ROSEN, D.W. Roadmap for Additive Manufacturing, University of Texas at Austin, Austin TX, 2009.

BRANDL, E. et al. Deposition of Ti-6Al-4V using laser and wire, part I: Microstructural properties of single beads. Surface \& Coatings Technology. p. 1120-1129. ago. 2011 (a).

BRANDL, E. et al. Deposition of Ti-6Al-4V using laser and wire, part II: Hardness and dimensions of single beads. Surface \& Coatings Technology. p. 1130-1141. ago. 2011 (b).

BRANDL, E. et al. Mechanical properties of additive manufactured titanium (Ti-6Al-4V) blocks deposited by a solid-state laser and wire. Materials And Design. p. 4665-4675. jul. 2011 (c).

BRANDL, E.; GREITEMEIER, D. Microstructure of additive layer manufactured Ti-6Al-4V after exceptional post heat treatments. Materials And Design. p. 84-87. abr. 2012 (d). BRANDL, E.; SCHOBERTH, A.; LEYENS, C. Morphology, microstructure, and hardness of titanium (Ti-6Al-4V) blocks deposited by wire-feed additive layer manufacturing (ALM). Materials Science And Engineering A. p. 295-307. nov. 2012 (e). CLARK, D.; BACHE, M.R.; WHITTAKER, M.T. Shaped metal deposition of a nickel alloy for aero engine applications. Journal of Materials Processing Technology, p.439-448, 2008.

DINDA, G.P.; DASGUPTA, A.K.; MAZUMDER, J. Laser aided direct metal deposition of Inconel 625 superalloy: Microstructural evolution and thermal stability. Materials Science and Engineering A, p.98-104, 2009.

D'OLIVEIRA, A.S.C.M. et al., Evaluation of Ni-Al coating processed by plasma transferred arc. Surface Engineering, v.27, 
n. 4, p. 266-271, 2011.

FLOWERS, G. E. et al. Elevated-Temperature, PlasmaTransferred Arc Welding of Nickel-Base Superalloy Articles. US nº 6084196, 25 fev. 1998, 04 jul. 2000. . Disponível em: $<$ https://www.google.com/patents/US6084196>. Acesso em: 22 jan. 2014.

FRAZIER, E. W. Metal Additive Manufacturing: A Review. Journal of Materials Engineering and Performance, v.23, p. 1917-1928, jun. 2014

GIBSON, I.; STUCKER, B.; ROSEN, D. W. Additive Manufacturing Technologies: Rapid Prototyping to Direct Digital Manufacturing. New York: Springer, 2010.

HENDERSON, M.B. et al., Nickel-Based Superalloy Welding Practices for Industrial Gas Turbine Applications. Science and Technology of Welding and Joining, no. 1, 2004.

JHAVAR, S., JAIN, N.K., PAUL, C.P. Development of microplasma transferred arc ( $\mu$-PTA) wire deposition process for additive layer manufacturing applications. Journal of Materials Processing Technology, p.1102-1110, 2014.

KATOU, M. et al., Freeform fabrication of titanium metal and intermetallic alloys by three-dimensional micro welding. Materials and Design, p. 2093-2098, 2007.

LEVY, G.N.; SCHINDEL, R.; KRUTH, J.P. Rapid manufacturing and rapid tooling with layer manufacturing (LM) technologies, state of the art and future perspectives. CIRP Annals - Manufacturing Technology, v. 52, 2.ed. p.589-609, 2003.

LIU, S. et al. Real-time monitoring of laser hot-wire cladding of Inconel 625. Optics \& Laser Technology. p. 124-134. mar. 2014. MARTINA, F. et al., Investigation of the benefits of plasma deposition for the additive layer manufacture of Ti-6Al-4V. Journal of Materials Processing Technology, p. 1377-1386, 2012.

MURR, L. E. et al. Fabrication of Metal and Alloy Components by Additive Manufacturing: Examples of 3D Materials Science. Journal of Materials Research and Technology, p. 4254. abr. 2012 (a).

MURR, L. E. et al. Microstructures and Properties of 17-4 PH Stainless Steel Fabricated by Selective Laser Melting. Journal of Materials Research and Technology, p. 167-177. set. 2012 (b).

MURR, L. E. et al. Metal Fabrication by Additive Manufacturing Using Laser and Electron Beam Melting Technologies. Journal of Materials Research and Technology, p. 1-14. out. 2012 (c).

SAJJADI, S.A. et al., Microstructure evolution of highperformance Ni-base superalloy GTD-111 with heat treatment parameters. Journal of Materials Processing Technology, p. 376381, 2006.

SKIBA, T.; BAUFELD, B.; BIEST, O. Microstructure and Mechanical Properties of Stainless Steel Component Manufactured by Shaped Metal Deposition. ISIJ International, p. 1588-1591, 2009.

SU, C.Y. et al., Plasma transferred arc repair welding of the nickel-base superalloy IN-738LC. Journal of Materials Engineering and Performance, p.619-627, 1997.

SURYAKUMAR, S. et al., Weld bead modeling and process optimization in Hybrid Layered Manufacturing. ComputerAided Design, 4.ed. p.331-344, 2011.
STEEN, W. M.; MAZUMDER, J. Laser Material Processing. 4. ed. Berlin: Springer-Verlag Ltd, 2010.

ZHAO, H. et al., A 3D dynamic analysis of thermal behavior during single-pass multi-layer weld-based rapid prototyping. Journal of Materials Processing Technology, p. 488-495, 2011.

ZHAO, H. et al., Three-dimensional finite element analysis of thermal stress in single-pass multi-layer weld-based rapid prototyping. Journal of Materials Processing Technology, p. 276-285, 2012.

XIONG, J.; ZHANG, G.; Adaptive control of deposited height in GMAW-based layer additive manufacturing. Journal of Materials Processing Technology, p. 962-968, 2014.

YILMAZ, O.; GINDY, N.; GAO, J. A repair and overhaul methodology for aeroengine components. Robotics And Computer-integrated Manufacturing. p. 190-201. jul. 2010. 